\title{
An ISP and End-User Cooperative Intradomain Routing Algorithm
}

\author{
Ali Norouzi, Michael Howarth and Ning Wang \\ Centre for Communication Systems Research \\ University of Surrey \\ Guildford, Surrey, United Kingdom \\ \{a.norouzi, m.howarth,n.wang\}@ surrey.ac.uk
}

\begin{abstract}
The continuous growth in volume of Internet traffic, including VoIP, IPTV and user-generated content, requires improved routing mechanisms that satisfy the requirements of both the Internet Service Providers (ISPs) that manage the network and the end-users that are the sources and sinks of data. The objectives of these two players are different, since ISPs are typically interested in ensuring optimised network utilisation and high throughput whereas end-users might require a low-delay or a high-bandwidth path. In this paper, we present our UAESR (Utilisation-Aware Edge Selected Routing) algorithm, which aims to satisfy both players' demands concurrently by selecting paths that are a good compromise between the two players' objectives. We demonstrate by simulation that this algorithm allows both actors achieve their goals. The results support our argument that our cooperative approach achieves effective network resource engineering at the same time as offering routing flexibility and good quality of service to end-users.
\end{abstract}

Keywords-component: Intradomain; QoS; ISP; End-user; UAESR Algorithm; Conflict of Interests; Players; Maximum Link Utilisation (MLU); End-to-End (ETE) Delay.

\section{INTRODUCTION}

\section{A. Background}

Ongoing proliferation of Internet content that requires specific network quality of service such as maximum delay or minimum bandwidth is a warning for traditional routing protocols. Internet Service Providers' (ISPs') networks need to be more efficient in network resource utilisation, reliable in data distribution, and diverse in quality of service realisation. As a result, a number of researchers have tried to improve the performance of data distribution and retrieval in the Internet using "multipath routing" as one approach to incrementally change the current Internet architecture so that the total capacity of the network is used more efficiently. This will improve data delivery performance and capacity (by providing path diversity), as well as improving routing around congestion and failures in the network (reliability). At the same time, we consider it important that the end-user should have some degree of control over the quality of service provided by the network, rather than simply being forced to follow a single path, as is the case with today's routing protocols [1][2][3]. By introducing a modest amount of control for end-users we demonstrate that ISPs' responsibility for maintaining a working network is not compromised. However, the interactions between end-users and ISPs on how to optimise data dissemination and access in multipath routing from both user and network perspectives introduces some closely inter-related challenges:

\section{1) Network Resource Management}

Given the increasingly high-volume content demand by applications with large bandwidth requirements, the effective management of network resources becomes important. Despite this importance, the main intra-domain routing algorithm, Open Shortest Path First (OSPF) computes only one path between any source and destination and this path is independent of network utilisation. Some more popular links will be used as hops of many shortest paths (due to their relatively low link cost) and eventually become hotspots suffering from congestion (Fig. 1). These congested and overutilised links exist while other links remain underutilised, which show poor traffic balancing and resource management practice. This arises because of the single path routing, given that there is path diversity in today's well-connected networks.

Likewise, BGP (Border Gateway Protocol), as the current interdomain routing protocol, allows only a single path between any two domains to reach a particular destination address prefix. However, in case of a failure in the end-toend (ETE) path, it takes BGP in order of minutes to reconverge. This convergence delay can have significantly negative impacts on subsequent data flows, which have no other choice to route around the congested or failed link or node and avoid service deterioration (delay, jitter, loss). Like OSPF, BGP does not change its routing decision in the event of congestion.

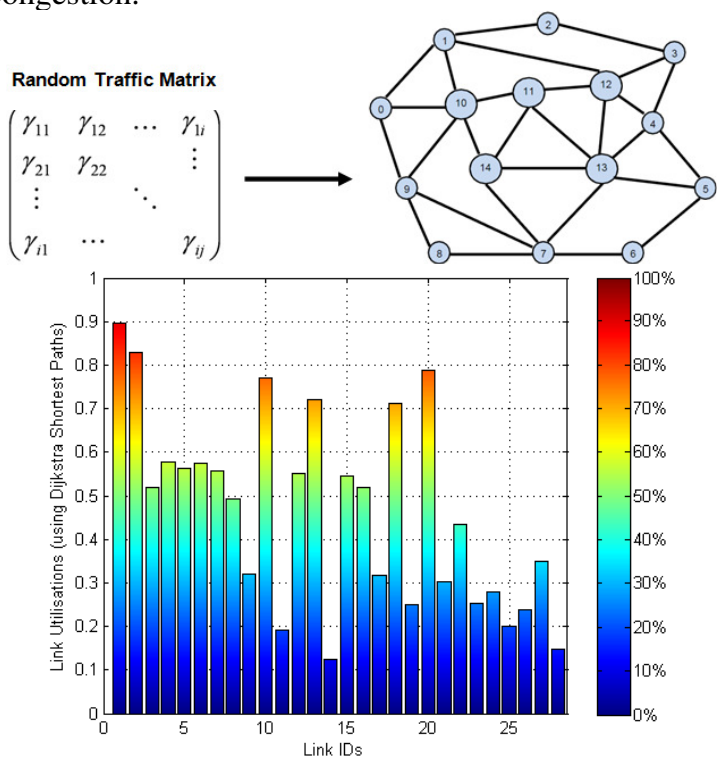

Figure 1. Unbalanced link utilisations caused by unfair distribution of a random traffic matrix across the Calle network [4] using shortest paths 


\section{2) Quality of Service ( $Q o S)$}

QoS support is an important feature of the Internet architecture. Different applications require different levels of service guarantee in order to function properly. This is particularly important for delay-sensitive and bandwidth intensive services, since their successful operation depends on the minimum QoS they receive. In spite of this, explicit QoS has failed to emerge in the Internet as an open ETE service. This is due to the fact that providing guaranteed QoS requires deployment of a complex and expensive QoS provisioning mechanism that does not have guaranteed increased revenue [1], and consequently ISPs lack the incentives to invest in QoS implementation.

\section{3) Control over Selection of Data Delivery Paths}

Currently, ISPs have full control over the ETE path. Packets of data take routes from source to destination precomputed by the network providers. Although a lot of data in the Internet is generated by the end-users, they do not have any control over their data delivery paths. The problem is if a flow faces congestion in the ETE path, the end-user cannot do anything to avoid it.

Clark et al. [1] point out this conflict in today's Internet and encourage a more open architecture that supports more choice as well as greater ability of all communication parties to express their preferences. As an example, they suggest end-user empowerment in ETE path selection to foster competition among ISPs to provide better services at lower costs and improve ETE performance and reliability [1][2]. The rationale for source controlled routing approaches is the ability of end-users to react to service disruptions even after noticing the QoS degradation, as a result of exclusive path selection performed by ISPs in conventional routing protocols. A game theory study of selfish routing (i.e. source routing and overlay routing approaches) [5] has shown that selfish routing can achieve close to optimal average latency but at the cost of increased congestion on some links. On the other hand, source routing makes the traffic engineering more difficult for providers, particularly for any intermediate ASes in an interdomain ETE path. Granting too much control to the end-users may result in traffic oscillation. To compute the paths, end-entities also depend on having (in principle) global knowledge of network topology. Getting these data to the end-nodes and keeping them updated with changes incurs a large overhead on the network [6].

\section{B. Related Work}

Various researchers have tried to address the issues outlined in Section I.A above, either fully or in part through deflection routing, multipath routing, or end-user empowerment in path selection.

OSR [7], AI-RON-E [8], congestion-triggered multipath routing algorithm [9], and SSPD [10] use deflection routing and hot potato routing mechanisms to deflect packets off the shortest ETE path and route around congested or failed links. However, lack of a centralised mechanism in these interdomain techniques may cause changes in ISPs' egress points and thus affect the extent of the region in which these algorithms are effective.
Other interdomain approaches try to overcome path failures with proactive construction of backup paths. BANANAS [11] provides multipath routing through partial upgrades of network nodes with flexible multipath-capable algorithms like $k$-shortest paths, all $k$-hop paths, or $k$-disjoint paths. GTSR [6] takes a game theoretic approach to devise alternate paths with minimum failure probability. YAMR [12] constructs multiple variations of BGP's default path, each of which assumes that a certain link in the default path is down. Path Splicing [13] combines multiple routing trees (slices) in the ETE path between the end-nodes by slight random perturbations of intradomain link weights and by storing $k$ best routes on the interdomain routers. The main drawback of these techniques is the overhead of storing extra forwarding entries in the forwarding table. The other issue is there might not be much QoS difference between the various alternative paths in some cases.

MIRO [3] suggests path multiplicity in interdomain paths through bilateral negotiations between neighbouring ASes based on a request-response query system. This however, comes at the cost of more signalling and memory overhead.

A number of proposals suggest a partial route concatenation approach to construct multiple paths as well as giving the user the power to choose the route segments. NIRA [2] realises user choice by giving the ability to create domain-level routes that consist of three sections, namely the sender side (gained via a Topology Information Propagation Protocol), the receiver side (obtained from a Name-to-Route Lookup Service), and the core (the network of Tier-1 providers). Platypus [14] uses an extra packet header to issue an authorisation stamp for a so-called waypoint, which is a resource principal responsible for traffic forwarding and billing functions. End-users then specify the waypoints to be traversed in the ETE path. Therefore, the ETE path is formed by concatenation of default paths between the waypoints. Pathlet routing [15] provides an interdomain source routing scheme over a virtual topology. Pathlet routing uses virtual nodes (vnodes) to represent arbitrary entities, such as an individual router, or an AS, or a region of the Internet. The sequence of vnodes which the ISP is willing to route the traffic through, forms one (or more, parallel) pathlet(s). The users then select and concatenate the disseminated pathlets to build ETE paths.

Other proposals (e.g. RAS [16], Path Brokering [17]) try to resolve the user-provider conflict by delegating the control of ETE route computation and forwarding infrastructure for customised routes to third parties. The third party entities act as retailers of ETE paths and have a global view of the Internet topology and the ability to compute user-desired paths and enforce them. They also hold payment mechanisms between the end-users and ISPs so that they do not have to negotiate directly. The drawback of these techniques is their disruptive approach which requires a lot of change in the control plane.

\section{Outline of Problem and Approach}

Most of the source controlled routing and multipath routing techniques discussed in the literature are interdomain and do not either consider the interactions between the end- 
user and the ISP in the intradomain or explain how the policy-compliant and user-desired paths are actually computed. This paper tries to address these gaps using a cooperative multipath routing approach for the intradomain.

Having noted the disadvantages of a purely network controlled routing paradigm in Section I.A, we also believe that a pure source controlled routing approach is not scalable, in the sense of a source (either a user or stub ISP) having full visibility of the entire Internet and the power to compute the ETE paths. However, no multipath routing algorithm can be a desirable solution without taking into account the requirements of both entities (ISPs and end-users). On the one hand, the end-user who knows best what QoS condition is suitable for its particular application is looking for ensured quality for its services. But the user does not know more than the ISP about global network conditions, and the power of path selection without any guidance from the ISP may result in sub-optimal route selection. On the other hand, the ISP, with a complete view of network conditions, is concerned about the balance of traffic across the network. However, the network is not aware of a user's application requirements and may not be able to provide improved service without a hint from the user side. Here is a conflict [1] in satisfying both needs, as one can affect the other negatively.

We argue that the above challenges can be addressed with a middle solution, which looks at both sides' benefits by shifting the routing control plane from a purely network controlled paradigm to a more cooperative approach between the ISPs and the end-users. The expected outcome is that this cooperative approach will lead to better network resource management and improved delivery of quality of service (e.g. ETE delay) to network users.

We suggest this tussle can be resolved if the end-users are presented by the ISP with a limited set of path options. The ISP therefore essentially selects a small set of paths that meet its own performance objectives, such as optimised utilisation or congestion avoidance. The end-user then selects one of these choices, based on its own requirements. In this way, the network traffic is balanced and the end-users benefit from good QoS. By comparison, in pure source routing, all possible paths are candidates for selection by the end-user; and in conventional shortest path routing, only one path is offered by the ISP.

This paper demonstrates the principle of the cooperative approach between ISPs and end-users and its ability to satisfy the requirements of both players. It is not, however, the objective of this paper to address implementation aspects such as mechanisms for routing enforcement.

Our novel algorithm (Utilisation-Aware Edge Selected Routing - UAESR) for optimising the performance metrics for both ISP and end-user is presented in Section II. The simulation setup and assumptions are explained in section III and simulation results are presented and discussed in section IV. Finally, we conclude the findings and discuss future work directions in section $\mathrm{V}$.

\section{UTILISATION-AwARE EDGE SELECTED ROUTING}

The Utilisation-Aware Edge Selected Routing (UAESR) algorithm tries to fulfil both of the two following objectives:
- From the ISP's perspective: minimise the maximum link utilisation in order to prevent congestion and balance the traffic across the network.

- From the end-user's perspective: select a path with either low delay, or high-bandwidth, or low loss rate, or other user-centric metric, to enable content to be transmitted across the Internet without service deterioration.

The term "end-user" should be taken to mean any entity at the edge of the network, for example, a network user, application software, or a neighbouring ISP.

UAESR works as follows:

- The network proactively computes a few least utilised paths between every source and destination router using a heuristic path computation shown as flowchart in Fig. 2. We assume the network knows the current link utilisations (averaged over some period) via a distributed monitoring system. The path computation mechanism prunes the most utilised links in the network topology graph one by one up to the point where there is no path between source and destination. It then puts back the last removed link to form the least utilised path (Fig. 3). For increased path diversity, removed paths are re-inserted in the topology graph one at a time, until there are a few paths between the two end-nodes. These paths are therefore those with the lowest values of maximum utilisation. A set of the best least utilised paths are saved for each source and destination router pair.

- The network presents the user with the pre-computed least utilised path(s) identified by the heuristic of Fig. 2 as well as the default shortest path (with least path cost). The end-user then chooses one, and sends its packets over this path (the precise mechanism for this is outside the scope of this paper). The end-user might select the path randomly, or might test the quality of path options with a probing method and then select one path on the basis of its desired QoS. However, the probing itself may cause some delay. Alternatively, depending on the implementation details, the network may be able to provide some of this QoS information to the end-user at the same time as giving the set of path options to it.

- The path computation is repeated regularly to update the previous paths and ensure their promised quality after regular changes in traffic load. The update frequency depends on the dynamics of the network.

\section{SimUlATION}

In order to evaluate the performance of the proposed algorithm, we implemented the time-based proactive UAESR algorithm and standard Shortest Path Dijkstra's algorithm (SP) in Java. Then, we conducted simulations with the following performance objectives:

- ISP-side: minimise the maximum link utilisation

- User-side: minimise the ETE delay.

It is important to note that UAESR is not to be considered as a replacement for shortest path routing, but as a complementary algorithm which offers least utilised paths for only a particular group of traffic flows (e.g. video streaming data), and is not for all of the traffic. We believe 
that this traffic split as a result will lead to more balanced traffic distribution throughout the network.

\section{A. Delay Modelling}

We assume a simple model for the link delay based on $\mathrm{M} / \mathrm{M} / 1$ queuing theory (Poisson Process) [18] in which the link delay $(\Delta)$ is calculated as a function of its utilisation $(\rho)$ :

$$
\Delta=\rho /(1-\rho)
$$

$\rho=\lambda / \mu$ wherein $\lambda$ is the traffic volume on the link and $\mu$ is the link capacity.

\section{B. Simulated Topology}

We used the test intradomain topology introduced by Calle [4] with 15 nodes and 28 links. This topology has been used in a number of studies. In our simulations, all links in Calle topology are assumed to have the same capacity (1000 bandwidth (BW) units). We also adopt the standard approach of setting the link weights to be inversely proportional to their capacities. Since we assume all links to have the same capacity in this paper, their link weights are the same and are set to one, i.e. our simulation implements shortest path routing by hop count.

\section{Simulation Setup}

- The simulation runs over 300 seconds and 60,000 random flows each of one bandwidth unit are generated between sources and destinations in the network. Each of these flows lasts for 30 seconds, so that we can test the network in steady state traffic conditions. The traffic flows start at random times (random traffic matrix), evenly spread over the simulation period.

The above numbers result in average network utilisation of about $45 \%$ according to the following formula (aggregate fraction of total network resources used):

$\left(\mathrm{BW}_{\text {flow }} \times \mathrm{No}_{\text {flows }} \times \mathrm{T}_{\text {flow }} \times\right.$ Avg Hops ETE SP $) /\left(\mathrm{T}_{\text {sim }} \times \mathrm{C}_{\text {net }}\right)(2)$ where $\mathrm{BW}_{\text {flow }}$ is the bandwidth of each flow, $\mathrm{No}_{\text {flows }}$ is the total number of flows, $T_{\text {flow }}$ is the duration of each flow,

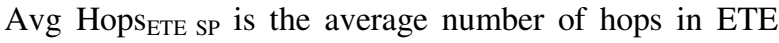
shortest paths, $\mathrm{T}_{\text {sim }}$ is the duration of simulation and the $\mathrm{C}_{\text {net }}$ is the overall network capacity, giving a mean network utilisation:

$$
(1 \times 60000 \times 30 \times 2.1) /(300 \times 28000) \approx 45 \%
$$

- Least utilised paths are updated regularly (the update frequency depends on the percentage of the total traffic that is carried by least utilised paths, the network traffic in terms of number of flows, and QoS metric). The least utilised path computation update frequency in the following simulations is set to 5 seconds because of high traffic generation rate in our simulation (200 ETE flows a second). In addition, when the least-utilised-path-directed fraction of traffic increases, more frequent path computations are needed to keep up with the dynamics of the network. This is due to the fact that the least utilised paths in our current implementation are not completely disjoint. Therefore, they can attract a lot of traffic during a very short time, which brings significant delay and congestion if the paths are not updated regularly.

- Every flow stays on the same path during its lifetime; once it is assigned to a path, the path does not change.

\section{Simulation Assumptions}

- We assume that a network monitoring system exists, which provides link utilisations to the network operator, for example using SNMP to retrieve measurements from the network averaged over some time. Therefore, the system uses real-time measurement of traffic volumes, and there is no prediction of traffic.

- Mechanisms are assumed to exist by which the network notifies the end-users of path options between source / destination (router) pair and the end-user notifies the network of its selection for different traffic flows.

- There is a central path computation entity in the network, which is responsible for computing least utilised paths between every source and destination.

- The paths might be enforced using a path setup mechanism such as MPLS LSPs, multi-topology routing, an optimised link weight setting or any other mechanism.

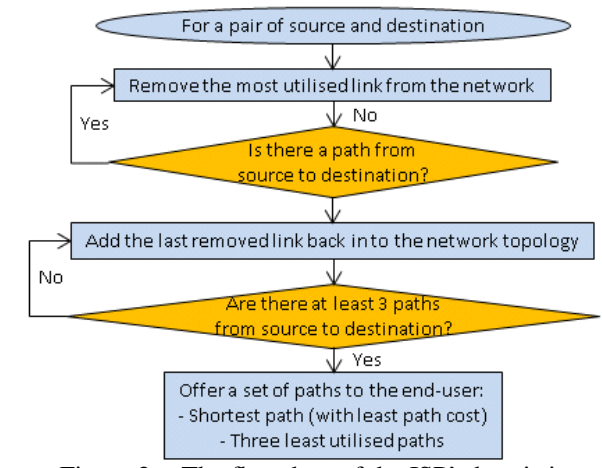

Figure 2. The flowchart of the ISP's heuristic
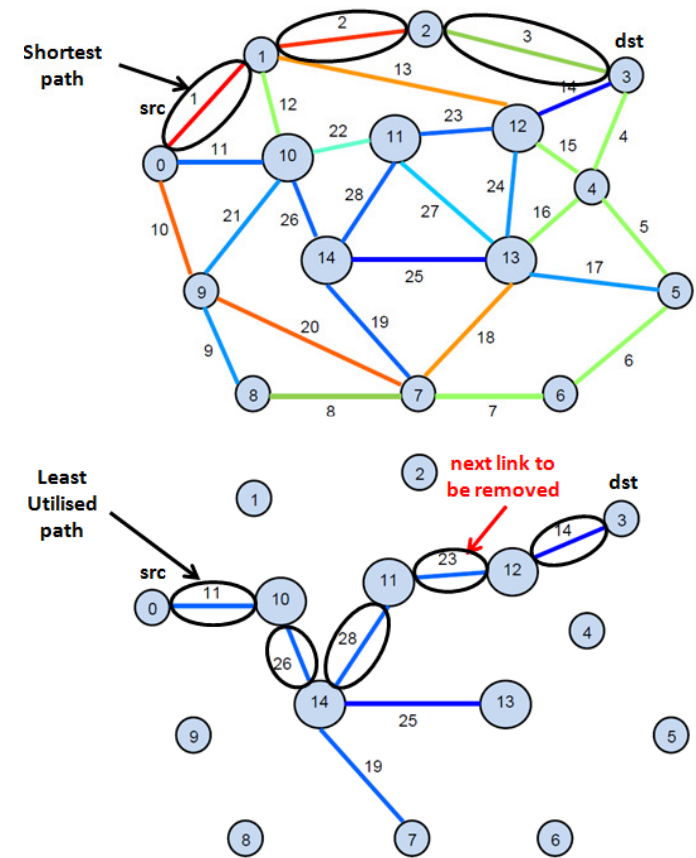

Figure 3. Example of a least utilised path between two nodes in the network: (a) the network in Fig. 1 with link numbers representing the link IDs and link colours corresponding to that of the link utilisation levels (b) The (single) least utilised path after applying the algorithm 


\section{RESULTS AND DISCUSSION}

In our results we compare four different ratios of enduser selected traffic: ratios of $25 \%-75 \%, 50 \%-50 \%, 75 \%$ $25 \%$ UAESR-SP directed traffic are compared with the case in which $100 \%$ of network traffic is routed through SPs ("UAESR" refers to the least utilised path computed by the UAESR algorithm and "SP" refers to standard Dijkstra's shortest path). The $100 \%$ SP case of course corresponds to today's best-effort Internet. We point out again that the UAESR algorithm is not intended here as a replacement for SP routing, but instead is a complementary algorithm which offers low utilisation paths for a particular group of traffic flows (e.g. streaming data) and we therefore imagine both routing algorithms coexisting.

In the simulation results in the remainder of this paper, the network calculates the three least utilised paths between every source and destination and presents these together with the shortest path to an end-user. Then, to simplify the simulation, we assume that the end-user always chooses the path that has the lowest ETE delay, possibly after probing all four candidates. In other words, UAESR optimises the path options based on link utilisations and the end-user chooses a path based on its desired metric, which for all simulation results presented in this paper is the lowest ETE delay.

The figures in this section show the following metrics as a function of time during the simulation.

\section{A. Mean Link Utilisation}

Fig. 4 shows that the average network load is higher in UAESR compared to shortest path. The reason is UAESR diverts the traffic off the shortest paths through more links, and, typically, the least utilised paths have more hops than the shortest paths. We note that the mean network utilisation rises as the percentage of flows that are carried on least utilised paths increases.

\section{B. Link Utilisation Variance}

Fig. 5 shows the variance of link utilisation across all links. The link utilisation variance is significantly lower when some flows are routed through least utilised paths. This shows that our algorithm balances out traffic very effectively, and displays the superiority of UAESR in traffic balancing capability. However, this improvement comes at the expense of higher average traffic in the network (Fig. 4).

\section{Maximum Link Utilisation}

Fig. 6 shows the maximum link utilisation across all network links, plotted as a function of time. Since the least utilised path traffic is routed through non-shortest paths (and actually through the least utilised links in the network), the maximum link utilisation decreases. This demonstrates the accomplishment of the UAESR algorithm in meeting the ISP's objective of minimising the maximum utilisation in the network and hence minimising congestion. Since the link delay (Eq. (1)) is highly sensitive to link utilisation, more lightly loaded links have a much lower delay. Consequently, by reducing the maximum link utilisation we achieve an overall decrease in the network ETE delay along a path (Fig. 8), in spite of the increase in the average link load (Fig. 4).
However, because UAESR generally increases the network load, this improvement becomes less noticeable as the UAESR-directed percentage of traffic increases.

\section{Mean Link Delay}

We now turn our attention to the end-users' principal objective. As stated at the start of Section IV, in the results presented here we assume that all end-users wish to minimise the ETE delay. We first therefore consider the mean of the delay on individual links, shown in Fig. 7, and then discuss the mean ETE delay in Fig. 8. In Fig. 7, since the traffic is more evenly carried by the network using least utilised paths, the utilisation of the most highly utilised links (i.e. those used by shortest path) has reduced. Therefore, the mean delay of all links has decreased. However, when the UAESR-directed fraction of all traffic flows increases to $50 \%$, its link delay curve almost equals that of $100 \%$ SP and in the case of $75 \%$ UAESR traffic, the link delay is worse than pure SP. This is because at very high levels of UAESR traffic, the network gets overloaded and severe delays occur on some links. We hypothesise that this might be more severe in our implementation of the least utilised path heuristic, in which the least utilised paths may not necessarily be sufficiently disjoint as a result of sharing particular low utilisation links. Thus, the negative impact of path overlapping may cause high volumes of traffic on certain links in a short time (a "rushing" phenomenon) and the least utilised links could become the most utilised links after some time.

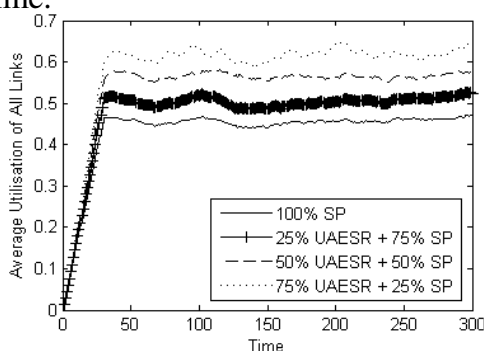

Figure 4. Mean Link Utilisation

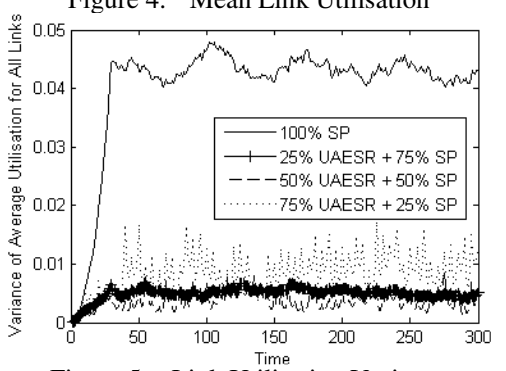

Figure 5. Link Utilisation Variance

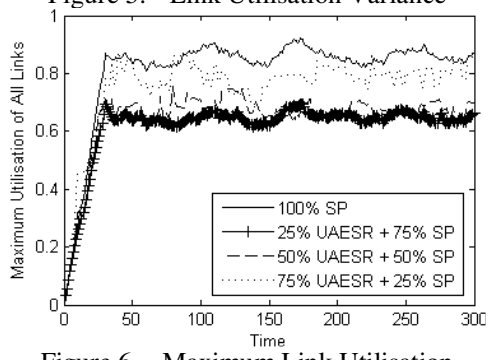

Figure 6. Maximum Link Utilisation 

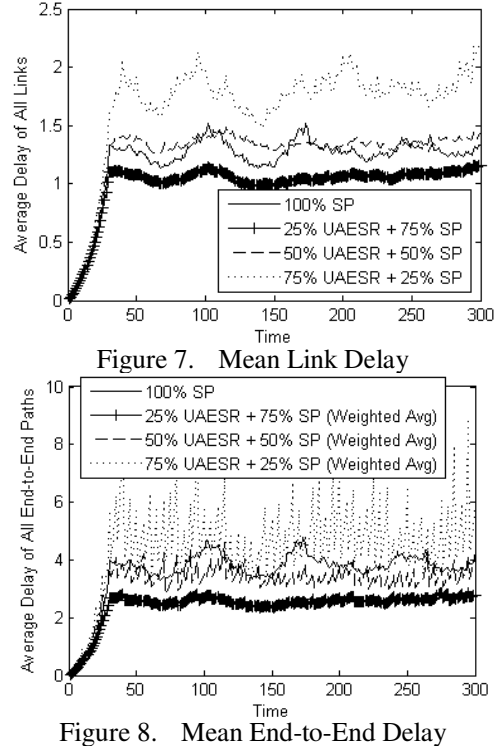

\section{E. Mean End-to-End Delay}

The mean ETE delay is shown in Fig. 8. The lower ETE delay when UAESR traffic comprises $25 \%$ or $50 \%$ of total traffic shows the improvement in packet delivery, and meets the end-user objective of low ETE delay. However, as with the mean link delay in Section IV.D, in the case of $75 \%$ UAESR traffic, the ETE delay fluctuates significantly, and is higher than the ETE delay in pure SP. This shows that the least utilised paths might not work effectively when implemented for a high fraction of total traffic. However, given that we conceive UAESR as co-existing with standard SP routing with the two algorithms being applied to a mix of traffic, we do not consider this to be a major drawback.

\section{F. Results Summary}

The results presented show that it is possible to improve the ETE path delay in the network whilst also reducing the MLU. This suggests that "win-win" solutions are available to satisfy the objectives of both actors (the ISPs and the endusers) at the same time without one negatively affect the other. An additional advantage of UAESR over other source routing algorithms is that although the least loaded paths are selected, the algorithm can be extended to incorporate additional ISP policies in to the path selection process if desired, since the end-user is only being offered a limited set of choices, all approved by the ISP.

\section{CONCLUSION}

A number of published studies hypothesise that user empowerment in the selection of ETE paths could help to boost data delivery performance. Nonetheless, this is only possible if users have influence over the selection of suitable path candidates, and not total control over the ETE path computation mechanism, since this might violate the ISPs' TE policies. Our simulation results have shown that UAESR achieves the following objectives for ISP and end-user:

- ISP: minimise the MLU, by balancing the network traffic

- End-user: deliver good quality paths with low ETE delay
Thus, if the operator chooses a short-list of paths and the end-user makes a final selection of those paths, the ETE delay is less than that obtained from the standard SP algorithms used in today's Internet, and the maximum link utilisation is lower than that obtained using SP. Therefore, the hypothesis is confirmed by the simulation results. Future work needs to consider the scalability, stability and dynamics of UAESR. Also further improvements to the details of the UAESR algorithm, to improve decoupling between the least utilised paths, may improve the utilisation and delay metrics.

\section{ACKNOWLEDGMENT}

This work was partially supported by the EU (IST) COMET project FP7-2010-ICT-248784.

\section{REFERENCES}

[1] D. Clark et al., "Tussle in cyberspace: defining tomorrow's Internet," IEEE/ACM Trans. Netw., vol. 13, no. 3, pp. 462-475, Jun. 2005.

[2] X. Yang, D. Clark, and A. W. Berger, "NIRA: A New Inter-Domain Routing Architecture," IEEE/ACM Trans. Netw., vol. 15, no. 4, pp. 775-788, Aug. 2007.

[3] W. Xu and J. Rexford, "MIRO: multi-path interdomain routing," SIGCOMM Comp. Comm. Rev, vol 36, no 4, pp.171-182, Aug 2006.

[4] E. Calle, J. Marzo, and A. Urra, "Protection performance components in MPLS networks," Comp. Comm., vol. 27, no. 12, July 2004, pp. 1220-1228, Jul. 2004.

[5] L. Qiu et al., "On selfish routing in internet-like environments," IEEE/ACM Trans. Netw., vol. 14, no. 4, pp. 725-738, Aug. 2006.

[6] C. Lim et al., "On the effectiveness of proactive path-diversity based routing for robustness to path failures," In Proc. 7th Int. IFIP-TC6 Networking Conf. AdHoc and Sensor Networks, Wireless Networks, Next Generation Internet, Springer-Verlag, Berlin, 2008, pp. 574-585.

[7] K. P. Gummadi et al., "Improving the reliability of internet paths with one-hop source routing," In Proc. 6th Conf. Symp. Opearting Systems Design \& Implementation, Berkeley, CA, 2004, pp. 13-13.

[8] Soon Hin Khor and A. Nakao, "AI-RON-E: Prophecy of one-hop source routers," In IEEE Global Telecommun. Conf., 2008, pp. 1-6.

[9] S. Sohn, B. L. Mark, and J. T. Brassil, "Congestion-triggered multipath routing based on shortest path information," In Proc. 15th Int. Conf. Comp. Comm. and Networks, 2006, pp. 191-196.

[10] X. Yang and D. Wetherall, "Source selectable path diversity via routing deflections," SIGCOMM Comp. Comm. Rev., vol. 36, no. 4, pp. 159-170, Aug. 2006.

[11] H. T. Kaur et al., "BANANAS: an evolutionary framework for explicit and multipath routing in the internet," SIGCOMM Comp. Comm. Rev., vol. 33, no. 4, pp. 277-288, Aug. 2003.

[12] I. Ganichev et al., "YAMR: yet another multipath routing protocol," SIGCOMM Comp. Comm. Rev., vol. 40, no. 5, pp. 13-19, Oct. 2010.

[13] M. Motiwala et al., "Path splicing," SIGCOMM Comput. Commun. Rev., vol. 38, no. 4, pp. 27-38, Aug. 2008.

[14] B. Raghavan, P. Verkaik, and A. C. Snoeren, "Secure and PolicyCompliant Source Routing," IEEE/ACM Trans. Netw., vol. 17, no. 3, pp. 764-777, Jun. 2009.

[15] P. B. Godfrey et al., "Pathlet routing," SIGCOMM Comp. Comm. Rev., vol. 39, no. 4, pp. 111-122, Aug. 2009.

[16] K. Lakshminarayanan, I. Stoica, and S. Shenker, "Routing as a service," UC Berkeley Tech. Rep. UCB/CSD-04-1327, 2004.

[17] J. R. Lane and A. Nakao, "Path brokering for end-host path selection: Toward a path-centric billing method for a multipath internet," In Proc. ACM CoNEXT Conf., Madrid, Spain, 2008.

[18] I. Adan and J. Resing, "M/M/1 queue," In Queueing theory, Dept. Math. Comput. Sci., Eindhoven Univ. of Tech., 2002, ch. 4, pp. 2942 , http://www.win.tue.nl/ iadan/queueing.pdf. 